\title{
ENDPOINT MAXIMAL AND SMOOTHING ESTIMATES FOR SCHRÖDINGER EQUATIONS
}

\author{
KEITH M. ROGERS AND ANDREAS SEEGER
}

\begin{abstract}
For $\alpha \in(0,1) \cup(1, \infty)$ we consider the initial value problem for the dispersive equation $i \partial_{t} u+(-\Delta)^{\alpha / 2} u=0$. We prove an endpoint $L^{p}$ inequality for the maximal function $\sup _{t \in[0,1]}|u(\cdot, t)|$ with initial values in $L^{p}$-Sobolev spaces, for $p \in(2+4 /(d+1), \infty)$. This strengthens the fixed time estimates due to Fefferman and Stein, and Miyachi. As an essential tool we establish sharp $L^{p}$ space-time estimates (local in time) for the same range of $p$.
\end{abstract}

\section{INTRODUCTION}

For $\alpha \in(0,1) \cup(1, \infty)$ we consider $L^{p}$ estimates for solutions to the initial value problem

$$
\left\{\begin{array}{l}
i \partial_{t} u+(-\Delta)^{\alpha / 2} u=0 \\
u(\cdot, 0)=f
\end{array}\right.
$$

The case $\alpha=2$ corresponds to the Schrödinger equation. We will not consider $\alpha=1$ which corresponds to the wave equation and exhibits different mathematical features.

When $f$ is a Schwartz function, the solution can be written as $u(x, t)=U_{t}^{\alpha} f(x)$, where

$$
\widehat{U_{t}^{\alpha}} f(\xi)=e^{i t|\xi|^{\alpha}} \widehat{f}(\xi)
$$

with $\widehat{f}(\xi)=\int f(y) e^{-i\langle y, \xi\rangle} d y$ as the definition of the Fourier transform. The sharp endpoint $L^{p}$-Sobolev bounds for fixed $t$ are due to Fefferman and Stein [10] and Miyachi [14]. Their result states that for any compact time interval $I$ and any $p \in(1, \infty)$,

$$
\sup _{t \in I}\left\|U_{t}^{\alpha} f\right\|_{L^{p}\left(\mathbb{R}^{d}\right)} \leqslant C_{I, p}\|f\|_{L_{\beta}^{p}\left(\mathbb{R}^{d}\right)}, \quad \frac{\beta}{\alpha}=d\left|\frac{1}{2}-\frac{1}{p}\right| ;
$$

this is sharp with respect to the regularity index $\beta$ and can also be deduced from certain endpoint versions of the Hörmander multiplier theorem ([1], [19]).

We strengthen the fixed time estimates as follows.

Theorem 1.1. Let $p \in\left(2+\frac{4}{d+1}, \infty\right)$ and $\alpha \neq 1$. Then, for any compact time interval $I$,

$$
\left\|\sup _{t \in I}\left|U_{t}^{\alpha} f\right|\right\|_{L^{p}\left(\mathbb{R}^{d}\right)} \leqslant C_{I, p}\|f\|_{L_{\beta}^{p}\left(\mathbb{R}^{d}\right)}, \quad \frac{\beta}{\alpha}=d\left(\frac{1}{2}-\frac{1}{p}\right) .
$$

This implies pointwise convergence results; indeed we shall prove a little more, namely if $\chi \in C_{c}^{\infty}(\mathbb{R})$ then the function $t \mapsto \chi(t) U_{t}^{\alpha} f(x)$ belongs to the Besov space $B_{1 / p, 1}^{p}(\mathbb{R})$, for almost every $x \in \mathbb{R}^{d}$. In particular these functions are continuous (for almost every $x$ ) and therefore this implies almost everywhere convergence to the initial datum as $t \rightarrow 0$.

2000 Mathematics Subject Classification. 42B15, 35B65.

Key words and phrases. Schrödinger equation, dispersive equations, pointwise convergence, maximal functions, smoothing, space time regularity.

The first author was supported by MEC project MTM2007-60952 and UAM-CM project CCG07UAM/ESP-1664. The second author was supported in part by NSF grant DMS 0652890. 
Our maximal function result is closely related to certain space-time estimates which improve the regularity index. The first such bounds are due to Constantin and Saut [7], Sjölin [21], and Vega [27] who showed that better $L^{2}$ regularity properties hold locally when $\alpha \in(1, \infty)$; namely, if $f \in L_{-(\alpha-1) / 2}^{2}\left(\mathbb{R}^{d}\right)$ then $u \in L_{\text {loc }}^{2}\left(\mathbb{R}^{d+1}\right)$. However, it is not possible to replace the $L^{2}$-norms over compact sets by $L^{2}$-norms which are global in space. This is known as the local smoothing phenomenon. For functions in $L^{2}$-Sobolev spaces the various local and global problems for smoothing and for maximal operators have received a lot of attention, starting with [4]. We do not have a contribution to the $L^{2}$-Sobolev problems but rather consider corresponding questions with initial data in $L^{p}$-Sobolev spaces for $p>2$, with $p$ not close to 2 .

In [16] the first author considered $L^{p}$ regularity estimates which are global in space but involve an integration over a compact time interval $I$,

$$
\left(\int_{I}\left\|U_{t}^{\alpha} f\right\|_{p}^{p} d t\right)^{1 / p} \leqslant C_{I}\|f\|_{L_{\beta}^{p}\left(\mathbb{R}^{d}\right)} .
$$

This question was motivated by the similar (although deeper) question for the wave equation (cf. [22], [28]). In [16], it was proven that (1.3) holds for $\alpha=2$ when $p>2+4 /(d+1)$ with $\beta / 2>d(1 / 2-1 / p)-1 / p$. We remark that smoothing results of this type could also be deduced from square-function estimates related to Bochner-Riesz multipliers such as in [2], [6], [18] and [13] however these arguments do not apply when $d=1$, and in dimensions $d \geqslant 2$ they are currently limited to the smaller range $p>2+4 / d$.

The $L^{p}$ smoothing result in [16] was obtained from an $L^{p} \rightarrow L^{p}$ estimate for the adjoint Fourier restriction (or 'extension') operator associated to the paraboloid, and the range $p>2+\frac{4}{d+1}$ corresponds to the known range of $L^{q} \rightarrow L^{p}$ bounds for the extension operator; see [8], [11] and [29] for the sharp bounds when $d=1$, and [24] for the best known partial results for $d \geqslant 2$. The reduction in [16] to the extension estimate used the explicit formula

$$
e^{i t \Delta} f(x)=\frac{1}{(4 \pi i t)^{d / 2}} \int e^{i|x-y|^{2} / 4 t} f(y) d y
$$

together with a 'completing of the square' trick; see [3] for a similar argument. Unfortunately this reasoning is not available when $\alpha \neq 2$.

We generalize to all $\alpha \neq 1$, and establish the endpoint regularity result.

Theorem 1.2. Let $p \in\left(2+\frac{4}{d+1}, \infty\right)$ and $\alpha \neq 1$. Then, for any compact time interval $I$,

$$
\left(\int_{I}\left\|U_{t}^{\alpha} f\right\|_{p}^{p} d t\right)^{1 / p} \leqslant C_{I}\|f\|_{L_{\beta}^{p}\left(\mathbb{R}^{d}\right)}, \quad \frac{\beta}{\alpha}=d\left(\frac{1}{2}-\frac{1}{p}\right)-\frac{1}{p} .
$$

In Theorem 4.1 below we formulate a slightly improved version of this result which can also be used to prove Theorem 1.1.

We mention an application in one spatial dimension where we obtain sharp estimates for the initial value problem for the Airy equation

$$
u_{t}+u_{x x x}=0 .
$$

For $f:=u(\cdot, 0)$ a Schwartz function, we can write $u(\cdot, t)=U_{t}^{3} P_{+} f+U_{-t}^{3} P_{-} f$, where $P_{+}$and $P_{-}$are the projection operators with Fourier multipliers $\chi_{(0, \infty)}$ and $\chi_{(-\infty, 0)}$, respectively. Thus, for initial values in $L_{\beta}^{p}$ the solution of (1.4) satisfies the sharp bound

$$
\|u\|_{L^{p}(\mathbb{R} \times[-T, T])} \leqslant C_{T}\|u(\cdot, 0)\|_{L_{\beta}^{p}(\mathbb{R})}, \quad \beta=\frac{3(p-4)}{2 p}, \quad 4<p<\infty,
$$


and if $u(\cdot, 0) \in L_{\varepsilon}^{p}(\mathbb{R})$ for any $\epsilon>0$ with $2<p \leqslant 4$, then $u \in L^{p}(\mathbb{R} \times[-T, T])$.

The proofs will be based on the bilinear adjoint restriction theorem for elliptic surfaces due to Tao [24]. In $\S 3$, having discussed the necessary conditions in $\S 2$, we combine Tao's theorem with a variation of a localization technique employed in [9] to prove $L^{p}$ estimates for some oscillatory integrals with elliptic phases; this yields the smoothing estimate for functions which are frequency supported in an annulus. In $\S 4$, we extend to the general case by decomposing the Fefferman-Stein sharp function; here we use a variant of an argument in [19].

Notation. Throughout, $c$ and $C$ will denote positive constants that may depend on the dimensions, exponents or indices of the Sobolev spaces, or the parameter $\alpha$, but never on the functions. Such constants are called admissible and their values may change from line to line. We shall mostly use the notation $A \lesssim B$ if $A \leqslant C B$ for an admissible constant $C$. We may sometimes indicate the dependence on a specific parameter $c$ by using the notation $\lesssim_{c}$. We write $A \approx B$ if $A \lesssim B$ and $B \lesssim A$.

\section{NeCESSARY CONDITIONS}

Let $\theta$ be a nonnegative and smooth function supported in $\left\{2^{-1}<|\xi|<2\right\}$ and equal to 1 in $\left\{2^{-1 / 2}<|\xi|<2^{1 / 2}\right\}$. For large $\lambda$, we consider initial data $f_{\lambda}$ defined by $\widehat{f}_{\lambda}(\xi)=$ $e^{-i|\xi|^{\alpha}} \theta\left(\lambda^{-1} \xi\right)$ and note that, by a change of variables,

$$
f_{\lambda}(x)=\left(\frac{\lambda}{2 \pi}\right)^{d} \int \theta(\xi) e^{i\left(\langle\lambda x, \xi\rangle-\lambda^{\alpha}|\xi|^{\alpha}\right)} d \xi
$$

Thus $\left|f_{\lambda}(x)\right| \lesssim \lambda^{d-\frac{d \alpha}{2}}$, by the method of stationary phase (keeping in mind that $\alpha \neq 1$ ). On the other hand, when $|x| \gg \lambda^{\alpha-1}$, by repeated integration by parts, there exists constants $C_{N}$ such that $\left|f_{\lambda}(x)\right| \leqslant C_{N}\left(|x| \lambda^{1-\alpha}\right)^{-N}$ for all $N \in \mathbb{N}$. Combining the two bounds, we see that

$$
\left\|f_{\lambda}\right\|_{L_{\beta}^{p}\left(\mathbb{R}^{d}\right)} \approx \lambda^{\beta}\left\|f_{\lambda}\right\|_{L^{p}\left(\mathbb{R}^{d}\right)} \lesssim \lambda^{d-\frac{d \alpha}{2}+\frac{d(\alpha-1)}{p}+\beta} .
$$

Next we consider $U_{t}^{\alpha} f_{\lambda}$ and compute

$$
\left|U_{t}^{\alpha} f_{\lambda}(x)\right|=\left|\left(\frac{\lambda}{2 \pi}\right)^{d} \int_{\mathbb{R}^{d}} \theta(\xi) e^{i\left(\langle\lambda x, \xi\rangle+\lambda^{\alpha}(t-1)|\xi|^{\alpha}\right)} d \xi\right|,
$$

so when $|x| \leqslant(10 \lambda)^{-1}$ and $|t-1| \leqslant\left(10 \lambda^{\alpha}\right)^{-1}$, we have $\left|U_{t}^{\alpha} f_{\lambda}(x)\right| \geqslant c \lambda^{d}$ for some positive constant $c$. Thus,

$$
\left(\int_{1-\left(10 \lambda^{\alpha}\right)^{-1}}^{1}\left\|U_{t}^{\alpha} f_{\lambda}\right\|_{p}^{p} d t\right)^{1 / p} \geqslant C \lambda^{d-\frac{d+\alpha}{p}} .
$$

Comparing this with the upper bound for $\left\|f_{\lambda}\right\|_{L_{\beta}^{p}\left(\mathbb{R}^{d}\right)}$, and letting $\lambda \rightarrow \infty$, we see that $\beta / \alpha \geqslant d(1 / 2-1 / p)-1 / p$ is a necessary condition for (1.3) to hold when $\alpha \neq 1$.

Note that alternatively one can argue that by Sobolev embedding any improvement in the smoothing would give a better fixed time estimate than the sharp known bounds in [10], [14], which is impossible.

The range $p>2+4 /(d+1)$ for the smoothing estimate in Theorem 1.2 is sharp for $d=1$, and for $d \geqslant 2$ it is conceivable that it holds for $p>2+2 / d$, see [16].

For Theorem 1.1 however our range may not be sharp even in one dimension. We can say that the maximal estimate (1.2) cannot hold when $p<2+1 / d$. This follows from the necessary condition $\beta / \alpha \geqslant 1 / 2 p$ which we now show, modifying a calculation in [17]. 
Let $\chi$ be a nonnegative and smooth function supported in $(-\varepsilon, \varepsilon)$ where $\varepsilon$ will be small depending only on $\alpha$. Let $e_{1}=(1,0, \ldots, 0)$ and define

$$
g_{\lambda}(x)=\frac{1}{(2 \pi)^{d}} \int \chi\left(\lambda^{\frac{\alpha-2}{2}}\left|\xi+\lambda e_{1}\right|\right) e^{i\langle x, \xi\rangle} d \xi
$$

Then immediately

$$
\left\|g_{\lambda}\right\|_{L_{\beta}^{p}} \lesssim \lambda^{\beta+\frac{d(\alpha-2)}{2}\left(\frac{1}{p}-1\right)}
$$

Now

$$
\begin{aligned}
U_{t}^{\alpha} g_{\lambda}(x) & =\frac{1}{(2 \pi)^{d}} \int \chi\left(\lambda^{\frac{\alpha-2}{2}}\left|\xi+\lambda e_{1}\right|\right) e^{i\left(\langle x, \xi\rangle+t|\xi|^{\alpha}\right)} d \xi \\
& =\frac{1}{(2 \pi)^{d}} \int \chi\left(\lambda^{\frac{\alpha-2}{2}}|h|\right) e^{i \phi_{\lambda}(x, t, h)} d h
\end{aligned}
$$

where $\phi_{\lambda}(x, t, h)=t \lambda^{\alpha}\left|-e_{1}+h / \lambda\right|^{\alpha}+\left\langle x,-\lambda e_{1}+h\right\rangle$. A Taylor expansion gives for $|h| \ll \lambda$

$$
\phi_{\lambda}(x, t, h)=t \lambda^{\alpha}-x_{1} \lambda+\left\langle x-t \alpha \lambda^{\alpha-1} e_{1}, h\right\rangle+O\left(\lambda^{\alpha-2} h^{2}\right)
$$

where the implicit constants in the error term depend on $\alpha$. The error term in the phase is $\ll 1$ on the support of the cutoff function (provided that $\varepsilon$ is sufficiently small).

Let $0<c \ll \alpha$ and let $R$ be the rectangle where $0 \leqslant x_{1} \leqslant c \lambda^{\alpha-1}$, and $\left|x_{i}\right| \leqslant \lambda^{(\alpha-2) / 2}$ for $i=2, \ldots, d$. We define $t(x)=\alpha^{-1} \lambda^{1-\alpha} x_{1}$ for $x \in R$ so that $t(x) \in[0,1]$ for $x \in R$, and for $x \notin R$ we may choose any (measurable) $t(x) \in[0,1]$. Then for $x \in R$, we have $\left|U_{t(x)}^{\alpha} g_{\lambda}(x)\right| \geqslant c_{0} \lambda^{-d(\alpha-2) / 2}$ and thus

$$
\left\|\sup _{0 \leqslant t \leqslant 1}\left|U_{t}^{\alpha} g_{\lambda}\right|\right\|_{p} \geqslant\left\|U_{t(\cdot)}^{\alpha} g_{\lambda}\right\|_{p} \gtrsim \lambda^{\frac{\alpha-1}{p}+\frac{(\alpha-2)(d-1)}{2 p}-\frac{(\alpha-2) d}{2}} .
$$

Comparing with the upper bound for $\left\|g_{\lambda}\right\|_{L_{\beta}^{p}}$ leads to the condition $\beta / \alpha \geqslant 1 / 2 p$.

\section{3. $L^{p}$ ESTIMATES FOR OSCILLATORY INTEGRALS WITH ELLIPTIC PHASES}

In the sequel, we will rescale inequalities for $U_{t}^{\alpha}$ when acting on functions with compact frequency support. This process will give rise to the operator $S$ defined by

$$
S f(x, t) \equiv S_{\chi}^{\phi} f(x, t)=\frac{1}{(2 \pi)^{d}} \int \chi(\xi) e^{i t \phi(\xi)} \widehat{f}(\xi) e^{i\langle x, \xi\rangle} d \xi
$$

where $\chi \in C_{0}^{\infty}(\mathcal{U})$ and $\phi$ is elliptic; here a $C^{\infty}$ function $\phi$ on an open set $\mathcal{U}$ in $\mathbb{R}^{d}$ is called elliptic if for every $\xi \in \mathcal{U}$ the Hessian $\phi^{\prime \prime}$ is positive definite.

We ask for $L^{p}\left(\mathbb{R}^{d}\right) \rightarrow L^{p}\left(\mathbb{R}^{d} \times[0, \lambda]\right)$ bounds for $S$. Note that for $|t| \leqslant 1$ and $\chi \in C_{0}^{\infty}$ the function $\chi e^{i t \phi}$ is a Fourier multiplier of $L^{p}, 1 \leqslant p \leqslant \infty$, and consequently the question is only nontrivial for large $\lambda$.

Proposition 3.1. Let $p>2+\frac{4}{d+1}, \chi \in C_{0}^{\infty}(\mathcal{U})$, and let $\phi$ be an elliptic phase on $\mathcal{U}$. Then

$$
\|S f\|_{L^{p}\left(\mathbb{R}^{d} \times[-\lambda, \lambda]\right)} \lesssim \lambda^{d(1 / 2-1 / p)}\|f\|_{L^{p}\left(\mathbb{R}^{d}\right)} .
$$

The key ingredient will be Tao's bilinear estimate for the adjoint restriction operator [24] which applies to phases which are small perturbations of $|\xi|^{2} / 2$. We need to formulate more specific assumptions on the phases allowed and follow [25]. Let $N \geqslant 10 d$. We say $\phi:[-2,2]^{d} \rightarrow \mathbb{R}$ is a phase of the class $\Phi(N, A)$ if $\left|\partial_{x_{j}}^{\alpha_{j}} \phi(x)\right| \leqslant A$ for all $x \in[-2,2]^{d}$ and all $\left|\alpha_{j}\right| \leqslant N$, where $j=1, \ldots, d$. To add an ellipticity condition we say that $\phi$ is of class 
$\Phi_{\text {ell }}(\varepsilon, N, A)$ if $\phi(0)=\nabla \phi(0)=0$, and if for all $x \in[-2,2]^{d}$ the eigenvalues of the Hessian $\phi^{\prime \prime}(x)$ lie in $[1-\varepsilon, 1+\varepsilon]$.

We define the adjoint restriction operator $\mathcal{E} \equiv \mathcal{E}^{\phi}$ by

$$
\mathcal{E} h(x, t)=\int_{[-2,2]^{d}} e^{i(\langle x, \xi\rangle+t \phi(\xi))} h(\xi) d \xi
$$

so that $S f=(2 \pi)^{-d} \mathcal{E} \widehat{f}$, where $\mathcal{U}=(-2,2)^{d}$. Now Tao's theorem can be stated as follows. Suppose $p>2+\frac{4}{d+1}$. Then there exists an $N$ (depending on $d$ and $p$ ) and for $A \geqslant 1$ there exists $\varepsilon=\varepsilon(A, N, d, p)>0$ so that the following holds for $\phi \in \Phi(\varepsilon, N, A)$ : For all pairs of $L^{2}$ functions $h_{1}, h_{2}$ so that $\operatorname{dist}\left(\operatorname{supp}\left(h_{1}\right)\right.$, supp $\left.\left(h_{2}\right)\right) \geqslant c>0$ the inequality

$$
\left\|\mathcal{E} h_{1} \mathcal{E} h_{2}\right\|_{p / 2} \lesssim_{c}\left\|h_{1}\right\|_{2}\left\|h_{2}\right\|_{2}, \quad p>2+\frac{4}{d+1},
$$

holds. In what follows we fix $N, A$ and $\varepsilon$ for which Tao's theorem applies. The constants may all depend on these parameters.

Lemma 3.2. Let $p>2+\frac{4}{d+1}$, let $B_{1}, B_{2} \subset[-1,1]^{d}$ be balls so that $\operatorname{dist}\left(B_{1}, B_{2}\right) \geqslant c$, and let $\phi \in \Phi_{\text {ell }}(\varepsilon, N, A)$. Then for $f, g$ with supp $\widehat{f} \subset B_{1}$, supp $\widehat{f} \subset B_{2}$,

$$
\|S f S g\|_{L^{p / 2}\left(\mathbb{R}^{d} \times[0, \lambda]\right)} \lesssim c, p \lambda^{d(1-2 / p)}\|f\|_{L^{p}\left(\mathbb{R}^{d}\right)}\|g\|_{L^{p}\left(\mathbb{R}^{d}\right)}
$$

Proof. Let $C_{0}=10\left(1+\max _{\xi \in[-2,2]^{d}}|\nabla \phi(\xi)|\right)$, and let $\eta_{1}, \eta_{2} \in C_{0}^{\infty}$ be supported in $(-2,2)^{d}$ so that $\eta_{1}(\xi)=1$ on $B_{1}$ and $\eta_{2}\left(\xi_{2}\right)=1$ on $B_{2}$. Moreover assume that $\eta_{1}$ and $\eta_{2}$ are supported on slightly larger concentric balls $\widetilde{B}_{1}, \widetilde{B}_{2}$ with the property that $\operatorname{dist}\left(\widetilde{B}_{1}, \widetilde{B}_{2}\right) \geqslant c / 2$. We also set

$$
P_{i} f=\mathcal{F}^{-1}\left[\eta_{i} \widehat{f}\right], \quad i=1,2 .
$$

Let $K_{t}^{i}=\mathcal{F}^{-1}\left[e^{i t \phi} \eta_{i} \chi\right]$, for $i=1,2$, so that

$$
S_{i} f(x, t):=S P_{i} f(x, t)=K_{t}^{i} * f(x) .
$$

Then $S f S g=S_{1} f S_{2} g$. We first note that for all $t \in[-\lambda, \lambda]$

$$
\left|K_{t}^{i}(x)\right| \lesssim|x|^{-N}, \quad \text { if }|x| \geqslant C_{0} \lambda
$$

This follows by a straightforward $N$-fold integration by parts, which uses the inequality $\left|\nabla_{\xi}(\langle x, \xi\rangle+t \phi(\xi))\right| \geqslant|x| / 2$ if $|x| \geqslant C_{0} \lambda,|t| \leqslant \lambda$.

Now let $\mathcal{Q}(\lambda)$ be a tiling of $\mathbb{R}^{d}$ by cubes of sidelength $\lambda$, and for each $Q \in \mathcal{Q}(\lambda)$ let $Q_{*}$ denote the enlarged cube with sidelength $2 C_{0} \lambda$, with the same center as $Q$. For each cube we split each function into a part supported in $Q_{*}$ and a part supported in its complement. Thus we can write

$$
\|S f S g\|_{L^{p / 2}\left(\mathbb{R}^{d} \times[0, \lambda]\right)}^{p / 2}=I+I I+I I I+I V
$$


where

$$
\begin{gathered}
I=\sum_{Q \in \mathcal{Q}(\lambda)}\left\|S_{1}\left[f \chi_{Q_{*}}\right] S_{2}\left[g \chi_{Q_{*}}\right]\right\|_{L^{p / 2}(Q \times[0, \lambda])}^{p / 2}, \\
I I=\sum_{Q \in \mathcal{Q}(\lambda)}\left\|S_{1}\left[f \chi_{Q_{*}}\right] S_{2}\left[g \chi_{\mathbb{R}^{d} \backslash Q_{*}}\right]\right\|_{L^{p / 2}(Q \times[0, \lambda])}^{p / 2}, \\
I I I=\sum_{Q \in \mathcal{Q}(\lambda)}\left\|S_{1}\left[f \chi_{\mathbb{R}^{d} \backslash Q_{*}}\right] S_{2}\left[g \chi_{Q_{*}}\right]\right\|_{L^{p / 2}(Q \times[0, \lambda])}^{p / 2}, \\
I V=\sum_{Q \in \mathcal{Q}(\lambda)}\left\|S_{1}\left[f \chi_{\mathbb{R}^{d} \backslash Q_{*}}\right] S_{2}\left[g \chi_{\mathbb{R}^{d} \backslash Q_{*}}\right]\right\|_{L^{p / 2}(Q \times[0, \lambda])}^{p / 2} .
\end{gathered}
$$

The first term gives the main contribution and is estimated using Tao's theorem, i.e. (3.2). One obtains,

$$
\begin{aligned}
|I| & \leqslant \sum_{Q \in \mathcal{Q}(\lambda)}\left\|S P_{1}\left[f \chi_{Q_{*}}\right] S P_{2}\left[g \chi_{Q_{*}}\right]\right\|_{L^{p / 2}\left(\mathbb{R}^{d} \times \mathbb{R}\right)}^{p / 2} \lesssim c \sum_{Q}\left\|P_{1}\left[f \chi_{Q_{*}}\right]\right\|_{2}^{p / 2}\left\|P_{2}\left[g \chi_{Q_{*}}\right]\right\|_{2}^{p / 2} \\
& \lesssim \sum_{Q}\left\|f \chi_{Q_{*}}\right\|_{2}^{p / 2}\left\|g \chi_{Q_{*}}\right\|_{2}^{p / 2} \lesssim\left(\sum_{Q}\left\|f \chi_{Q_{*}}\right\|_{2}^{p}\right)^{1 / 2}\left(\sum_{Q}\left\|g \chi_{Q_{*}}\right\|_{2}^{p}\right)^{1 / 2} .
\end{aligned}
$$

By Hölder's inequality,

$$
\left(\sum_{Q}\left\|f \chi_{Q_{*}}\right\|_{2}^{p}\right)^{1 / p} \lesssim\left(\sum_{Q}\left|Q_{*}\right|^{p / 2-1}\left\|f \chi_{Q_{*}}\right\|_{p}^{p}\right)^{1 / p} \lesssim \lambda^{d(1 / 2-1 / p)}\|f\|_{p},
$$

and we have the same estimate for $g$. Thus $I^{2 / p} \lesssim_{c} \lambda^{d(1-2 / p)}\|f\|_{p}\|g\|_{p}$ which is the desired bound for the main term.

The corresponding estimates for $I I, I I I, I V$ are straightforward as we use (3.3) for the terms supported in $\mathbb{R}^{d} \backslash Q_{*}$. We examine $I I$ and begin with

$$
\begin{aligned}
|I I| & \leqslant \sum_{Q \in \mathcal{Q}(\lambda)}\left\|S_{1}\left[f \chi_{Q_{*}}\right]\right\|_{L^{p}(Q \times[0, \lambda])}^{p / 2}\left\|S_{2}\left[g \chi_{\mathbb{R}^{d} \backslash Q_{*}}\right]\right\|_{L^{p}(Q \times[0, \lambda])}^{p / 2} \\
& \leqslant\left(\sum_{Q \in \mathcal{Q}(\lambda)}\left\|S_{1}\left[f \chi_{Q_{*}}\right]\right\|_{L^{p}(Q \times[0, \lambda])}^{p}\right)^{1 / 2}\left(\sum_{Q \in \mathcal{Q}(\lambda)}\left\|S_{2}\left[g \chi_{\mathbb{R}^{d} \backslash Q_{*}}\right]\right\|_{L^{p}(Q \times[0, \lambda])}^{p}\right)^{1 / 2} .
\end{aligned}
$$

We use the trivial bound $\left\|S_{1} f(\cdot, t)\right\|_{p} \lesssim(1+|t|)^{d}\|f\|_{p}$ for $f$ replaced with $f \chi_{Q_{*}}$, so that the first factor in (3.4) is bounded by $\left(C \lambda^{d+1}\|f\|_{p}\right)^{p / 2}$. By (3.3) we get

$$
\begin{aligned}
\left(\sum_{Q \in \mathcal{Q}(\lambda)}\left\|S_{2}\left[g \chi_{\mathbb{R}^{d} \backslash Q_{*}}\right]\right\|_{L^{p}(Q \times[0, \lambda])}^{p}\right)^{1 / p} & \\
& \lesssim\left(\int_{-\lambda}^{\lambda} \int_{x \in \mathbb{R}^{d}}\left[\int_{|z| \geqslant \lambda}|z|^{-N}|g(x-z)| d z\right]^{p} d x d t\right)^{1 / p} \lesssim \lambda^{d+1-N}\|g\|_{p} .
\end{aligned}
$$

Hence $|I I|^{2 / p} \lesssim_{c} \lambda^{2(d+1)-N}\|f\|_{p}\|g\|_{p}$. As $N \geqslant 10 d$ this estimate is negligible. Because of symmetry $I I I$ is estimated by the same term. For the estimation of $I V$ we proceed in the same way but use (3.3) for both terms, the result is the (again negligible) bound $|I V|^{2 / p} \lesssim \lambda^{2(d+1-N)}\|f\|_{p}\|g\|_{p}$.

We now formulate an analogous result for functions with smaller frequency support and smaller separation. 
Lemma 3.3. Let $p>2+\frac{4}{d+1}$ and $\lambda^{1 / 2} \geqslant 2^{j} \geqslant 1$. Let $Q_{1}, Q_{2} \subset[-1,1]^{d}$ be cubes of side $2^{j} \lambda^{-1 / 2}$, so that $\operatorname{dist}\left(Q_{1}, Q_{2}\right) \geqslant c 2^{j} \lambda^{-1 / 2}$ and let $\phi \in \Phi_{\mathrm{ell}}(\varepsilon, N, A)$. Then for all $f$ and $g$ such that supp $\widehat{f} \subset Q_{1}$, supp $\widehat{f} \subset Q_{2}$,

$$
\|S f S g\|_{L^{p / 2}\left(\mathbb{R}^{d} \times[0, \lambda]\right)} \lesssim_{c} 2^{4 j\left(\frac{d}{2}-\frac{d+1}{p}\right)} \lambda^{\frac{2}{p}}\|f\|_{L^{p}\left(\mathbb{R}^{d}\right)}\|g\|_{L^{p}\left(\mathbb{R}^{d}\right)} .
$$

Proof. By finite partitions and the triangle inequality, we may suppose that $Q_{1}$ and $Q_{2}$ are balls of radius $2^{j} \lambda^{-1 / 2}$. We reduce matters to the statement in Lemma 3.2 by scaling. Let $\xi_{0}$ be the midpoint of the interval connecting the center of the balls. We change variables $\xi=\xi_{0}+\delta \eta$ where $\delta=2^{j} \lambda^{-1 / 2}$. Then a short computation shows that

$$
S^{\phi} f(x, t)=e^{i\left(\left\langle x, \xi_{0}\right\rangle+t \phi\left(\xi_{0}\right)\right)} S^{\psi} f_{*}\left(\delta\left(x+t \nabla \phi\left(\xi_{0}\right)\right), \delta^{2} t\right) \quad \text { where } f_{*}(y)=f\left(\delta^{-1} y\right) e^{i \delta^{-1}\left\langle y, \xi_{0}\right\rangle},
$$

and the phase $\psi$ is given by

$$
\psi(\eta)=\frac{1}{2} \int_{0}^{1}\left\langle\phi^{\prime \prime}\left(\xi_{0}+s \delta \eta\right) \eta, \eta\right\rangle d s
$$

The same consideration is applied to $S^{\phi} g$. Note that $\psi$ is elliptic (with estimates uniform in $\xi_{0}$ and $\delta$ ) and the frequency supports of $f_{*}$ and $g_{*}$ are now separated, independently of $\delta, j$ and $\lambda$. Thus we can apply Lemma 3.2 to obtain

$$
\begin{aligned}
\left\|S^{\phi} f S^{\phi} g\right\|_{L^{p / 2}\left(\mathbb{R}^{d} \times[0, \lambda]\right)} & =\delta^{-(d+2) /(p / 2)}\left\|S^{\psi} f_{*} S^{\psi} g_{*}\right\|_{L^{p / 2}\left(\mathbb{R}^{d} \times\left[0, \lambda \delta^{2}\right]\right)} \\
& \lesssim \delta^{-(2 d+4) / p}\left(\lambda \delta^{2}\right)^{d(1-2 / p)}\left\|f_{*}\right\|_{p}\left\|g_{*}\right\|_{p} \\
& \lesssim \delta^{2 d-4(d+1) / p} \lambda^{d(1-2 / p)}\|f\|_{p}\|g\|_{p} .
\end{aligned}
$$

As $\delta=2^{j} \lambda^{-1 / 2}$ the assertion follows.

We will also require the following lemma for when we have no frequency separation.

Lemma 3.4. Let $p \geqslant 1$, let $Q \subset[-1,1]^{d}$ be a cube of side $\lambda^{-1 / 2}$, and let $\phi \in \Phi(N, A)$. Then for all $f$ such that supp $\widehat{f} \subset Q$,

$$
\|S f(\cdot, t)\|_{L^{p}\left(\mathbb{R}^{d}\right)} \lesssim\|f\|_{L^{p}\left(\mathbb{R}^{d}\right)}, \quad|t| \leqslant \lambda .
$$

Proof. Let $\xi_{B}$ be the center of the cube $Q$, and let $\chi \in C_{0}^{\infty}$ so that $\chi(\xi)=1$ for $|\xi| \leqslant \sqrt{d}$. It suffices to show that $\chi\left(\lambda^{1 / 2}\left(\xi-\xi_{B}\right)\right) e^{i t \phi(\xi)}$ is a Fourier multiplier of $L^{p}$ for all $|t| \leqslant \lambda$, with bounds uniform in $t$. By modulation, translation and dilation invariance of the multiplier norm it suffices to check that $h(\cdot, t)$ defined by

$$
h(\eta, t)=\chi(\eta) e^{i t\left(\phi\left(\lambda^{-1 / 2} \eta+\xi_{B}\right)-\phi\left(\xi_{B}\right)-\left\langle\lambda^{-1 / 2} \eta, \nabla \phi\left(\xi_{B}\right)\right\rangle\right)},
$$

is a Fourier multiplier of $L^{p}$, uniformly in $|t| \leqslant \lambda$. However this follows since $\partial_{\eta}^{\alpha} h(\eta, t)=$ $O(1)$ for $|t| \leqslant \lambda$ as one can easily check.

Proof of Proposition 3.1. By a partition of unity and a compactness argument it suffices to show that for every $\xi_{0} \in \mathcal{U}$ there is a neighborhood $\mathcal{U}\left(\xi_{0}\right)$ so that the statement of the theorem holds with $\chi$ replaced by $\chi_{0} \in C_{0}^{\infty}$ supported in $\mathcal{U}\left(\xi_{0}\right)$. Now let $\mathcal{H}$ be the (symmetric) positive definite squareroot of $\phi^{\prime \prime}\left(\xi_{0}\right)$ and let

$$
\psi(\eta)=\varepsilon_{1}^{-2}\left(\phi\left(\xi_{0}+\varepsilon_{1} \mathcal{H}^{-1} \eta\right)-\phi\left(\xi_{0}\right)-\varepsilon_{1}\left\langle\mathcal{H}^{-1} \eta, \nabla \phi\left(\xi_{0}\right)\right\rangle\right)
$$

Then it suffices to show that $S^{\psi}$ (defined with the amplitude $\chi\left(\xi_{0}+\varepsilon_{1} \mathcal{H}^{-1} \eta\right)$ ) satisfies the asserted estimates, with a dependence on $\varepsilon_{1}$. If $\varepsilon_{1}$ is chosen sufficiently small then we 
have reduced matters to a phase function in $\Phi_{\text {ell }}(\varepsilon, N, A)$ with parameters for which Tao's theorem and therefore Lemma 3.3 applies.

We now return to our original notation and work with a phase function $\phi$ but assume now that $\phi \in \Phi_{\text {ell }}(\varepsilon, N, A)$; we may also assume that the amplitude function $\chi$ is smooth and supported in $\left[-(2 d)^{-10},(2 d)^{-10}\right]^{-d}$. We make a decomposition of the product $S f S g$ in terms of bilinear operators, localizing the frequency variables in terms of nearness to the diagonal in $(\xi, \eta)$-space; this is similar to arguments in [12], [20] and [25].

Let $\chi_{0}$ be a radial $C_{0}^{\infty}\left(\mathbb{R}^{d}\right)$ function so that $\chi_{0}(\omega)=1$ for $|\omega| \leqslant 8 d^{1 / 2}$ and so that supp $\chi_{0}$ is contained in $\left\{\omega:|\omega|<16 d^{1 / 2}\right\}$. Fix $\lambda>1$ and set

$$
\begin{aligned}
& \Theta_{0}(\xi, \eta)=\chi_{0}\left(\lambda^{1 / 2}(\xi-\eta)\right) \\
& \Theta_{j}(\xi, \eta)=\chi_{0}\left(\lambda^{1 / 2} 2^{-j}(\xi-\eta)\right)-\chi_{0}\left(2 \lambda^{1 / 2} 2^{-j}(\xi-\eta)\right), \quad j \geqslant 1,
\end{aligned}
$$

so that $\Theta_{0}$ is supported where $|\xi-\eta| \leqslant 16 d^{1 / 2} \lambda^{-1 / 2}$ and, $\Theta_{j}$ is supported in the region

$$
4 d^{1 / 2} 2^{j} \lambda^{-1 / 2} \leqslant|\xi-\eta| \leqslant 16 d^{1 / 2} 2^{j} \lambda^{-1 / 2} .
$$

We may then decompose

$$
S f S g=\sum_{j \geqslant 0} \mathcal{B}_{j}[f, g]
$$

where

$$
\mathcal{B}_{j}[f, g](x, t)=\frac{1}{(2 \pi)^{2 d}} \iint e^{i\langle x, \xi+\eta\rangle} e^{i t(\phi(\xi)+\phi(\eta))} \Theta_{j}(\xi, \eta) \widehat{f}(\xi) \widehat{g}(\eta) d \xi d \eta
$$

Only values of $j \geqslant 0$ with $2^{j} \leqslant \lambda^{1 / 2}$ will be relevant, as otherwise $\mathcal{B}_{j}$ is identically zero. We will prove the estimate

$$
\left\|\mathcal{B}_{j}[f, g]\right\|_{p / 2} \lesssim\left\{\begin{array}{lr}
2^{4 j\left(\frac{d}{2}-\frac{d+1}{p}\right)} \lambda^{\frac{2}{p}}\|f\|_{p}\|g\|_{p}, & \frac{2(d+3)}{d+1}<p \leqslant 4, \\
2^{j\left(d-\frac{4}{p}\right)} \lambda^{\frac{d}{2}-\frac{2(d-1)}{p}}\|f\|_{p}\|g\|_{p}, & 4<p<\infty
\end{array}\right.
$$

and use this to bound

$$
\|S f\|_{L^{p}\left(\mathbb{R}^{d} \times[0, \lambda]\right)}=\left\|(S f)^{2}\right\|_{L^{p / 2}\left(\mathbb{R}^{d} \times[0, \lambda]\right)}^{1 / 2} \leqslant\left(\sum_{0 \leqslant j \leqslant \log _{2}\left(\lambda^{1 / 2}\right)}\left\|\mathcal{B}_{j}[f, f]\right\|_{p / 2}\right)^{1 / 2},
$$

and then sum a geometric series.

In order to prove (3.5), we decompose $\mathcal{B}_{j}$ into pieces on which we may apply Lemma 3.3. Let $\vartheta \in C_{0}^{\infty}\left(\mathbb{R}^{d}\right)$ a function supported in $[-3 / 5,3 / 5]^{d}$, equal to 1 on $[-2 / 5,2 / 5]^{d}$, and satisfying

$$
\sum_{n \in \mathbb{Z}^{d}} \vartheta(\xi-n)=1
$$

for all $\xi \in \mathbb{R}^{d}$. For $j \geqslant 0, n \in \mathbb{Z}^{d}$, define

$$
\beta_{j, n}(\xi)=\vartheta\left(\lambda^{1 / 2} 2^{-j} \xi-n\right)
$$

and, for $\left(n, n^{\prime}\right) \in \mathbb{Z}^{d} \times \mathbb{Z}^{d}$,

$$
\vartheta_{j, n, n^{\prime}}(\xi, \eta)=\Theta_{j}(\xi, \eta) \beta_{j, n}(\xi) \beta_{j, n^{\prime}}(\eta) .
$$

Observe that $\beta_{j, n}, \beta_{j, n^{\prime}}$ are supported in cubes $Q_{j, n}, Q_{j, n^{\prime}}$ which have sidelengths slightly larger than $\lambda^{-1 / 2} 2^{j}$, and that are centered at the points $\xi_{j, n}=\lambda^{-1 / 2} 2^{j} n$ and $\xi_{j, n^{\prime}}=$ $\lambda^{-1 / 2} 2^{j} n^{\prime}$, respectively. 
Now let

$$
\begin{aligned}
\Delta_{0} & =\left\{\left(n, n^{\prime}\right) \in \mathbb{Z}^{d} \times \mathbb{Z}^{d}:\left|n-n^{\prime}\right| \leqslant 18 d^{1 / 2}\right\}, \\
\Delta & =\left\{\left(n, n^{\prime}\right) \in \mathbb{Z}^{d} \times \mathbb{Z}^{d}: 2 d^{1 / 2} \leqslant\left|n-n^{\prime}\right| \leqslant 18 d^{1 / 2}\right\} .
\end{aligned}
$$

Then if $\vartheta_{0, n, n^{\prime}}$ is not identically zero then we necessarily have $\left(n, n^{\prime}\right) \in \Delta_{0}$ and if, for $j \geqslant 1$ the function $\vartheta_{j, n, n^{\prime}}$ is not identically zero then we necessarily have $\left(n, n^{\prime}\right) \in \Delta$. These statements follow by the definitions of our cutoff functions. Moreover,

$$
\operatorname{dist}\left(Q_{j, n}, Q_{j, n^{\prime}}\right) \leqslant 18 d^{1 / 2} 2^{j} \lambda^{-1 / 2} \quad \text { if }\left(n, n^{\prime}\right) \in \Delta_{0},
$$

and

$$
2^{-1} d^{1 / 2} 2^{j} \lambda^{-1 / 2} \leqslant \operatorname{dist}\left(Q_{j, n}, Q_{j, n^{\prime}}\right) \leqslant 18 d^{1 / 2} 2^{j} \lambda^{-1 / 2} \quad \text { if } j \geqslant 1 \text { and }\left(n, n^{\prime}\right) \in \Delta .
$$

For the application of Lemma 3.3 it is convenient to eliminate the cutoff $\Theta_{j}$ but still keep the separation of the supports of $\beta_{j, n}$ and $\beta_{j, n^{\prime}}$. Set, for $j \geqslant 1$,

$$
\widetilde{\mathcal{B}}_{j}[f, g](x, t)=\frac{1}{(2 \pi)^{2 d}} \iint e^{i\langle x, \xi+\eta\rangle} e^{i t(\phi(\xi)+\phi(\eta))} \sum_{n, n^{\prime} \in \Delta} \beta_{j, n}(\xi) \beta_{j, n^{\prime}}(\eta) \widehat{f}(\xi) \widehat{g}(\eta) d \xi d \eta
$$

and define $\widetilde{\mathcal{B}}_{0}[f, g]$ similarly by letting the $\left(n, n^{\prime}\right)$ sum run over $\Delta_{0}$. The reduction of the estimate for $\mathcal{B}_{j}$ to the estimate for $\widetilde{\mathcal{B}}_{j}$ is straightforward; by an averaging argument. Indeed, let $\chi_{1}=\chi_{0}-\chi_{0}(2 \cdot)$ and use the Fourier inversion formula

$$
\Theta_{j}(\xi, \eta)=\frac{1}{(2 \pi)^{d}} \int \widehat{\chi}_{1}(y) e^{i \lambda^{1 / 2} 2^{-j}\langle\xi-\eta, y\rangle} d y, \quad j \geqslant 1
$$

then

$$
\mathcal{B}_{j}[f, g]=\frac{1}{(2 \pi)^{d}} \int \widehat{\chi}_{1}(y) \widetilde{\mathcal{B}}_{j}\left[f_{-y}, g_{y}\right] d y
$$

where $f_{-y}(x)=f\left(x+\lambda^{1 / 2} 2^{-j} y\right)$ and $g_{y}(x)=g\left(x-\lambda^{1 / 2} 2^{-j} y\right)$. A similar formula holds for $j=0$, only then $\chi_{1}$ is replaced with $\chi_{0}$. Thus in order to finish the argument it is enough to show that $\left\|\widetilde{\mathcal{B}}_{j}[f, g]\right\|_{p / 2}$ is dominated by the right hand side of (3.5).

Define convolution operators $P_{j, n}$ by $\widehat{P_{j, n} f}=\beta_{j, n} \widehat{f}$. Note that for fixed $j$, each $\xi$ is contained in only a bounded number of the sets $Q_{j, n}+Q_{j, n^{\prime}}$. This implies, by interpolation of $\ell^{2}\left(L^{2}\right)$ with trivial $\ell^{1}\left(L^{1}\right)$ or $\ell^{\infty}\left(L^{\infty}\right)$ bounds that, for $j \geqslant 1, p \geqslant 2$,

$$
\begin{aligned}
\left\|\widetilde{\mathcal{B}}_{j}[f, g]\right\|_{L^{p / 2}\left(\mathbb{R}^{d} \times[0, \lambda]\right)} & \\
& \lesssim \max \left\{1,\left(\lambda^{1 / 2} 2^{-j}\right)^{d(1-4 / p)}\right\}\left(\sum_{n, n^{\prime} \in \Delta}\left\|S P_{j, n} f S P_{j, n^{\prime}} g\right\|_{L^{p / 2}\left(\mathbb{R}^{d} \times[0, \lambda]\right)}^{p / 2}\right)^{2 / p} .
\end{aligned}
$$

The analogous formula for $j=0$ holds if we replace $\Delta$ by $\Delta_{0}$. Notice that for all $j$,

$$
\left(\sum_{n}\left\|P_{j, n} f\right\|_{p}^{p}\right)^{1 / p} \lesssim\|f\|_{p}, \quad p \geqslant 2
$$

Now if $j=0$ we use Lemma 3.4 to estimate

$$
\begin{aligned}
\left\|S P_{0, n} f(\cdot, t) S P_{0, n^{\prime}} g(\cdot, t)\right\|_{L^{p / 2}\left(\mathbb{R}^{d}\right)} & \lesssim\left\|S P_{0, n} f(\cdot, t)\right\|_{p}\left\|S P_{0, n^{\prime}} g(\cdot, t)\right\|_{p} \\
& \lesssim\left\|P_{0, n} f\right\|_{p}\left\|P_{0, n^{\prime}} g\right\|_{p}
\end{aligned}
$$


hence, after integrating in $t$,

$$
\begin{aligned}
\left\|\widetilde{\mathcal{B}}_{0}[f, g]\right\|_{L^{p / 2}\left(\mathbb{R}^{d} \times[0, \lambda]\right)} & \lesssim \max \left\{1, \lambda^{d(1 / 2-2 / p)}\right\} \lambda^{2 / p}\left(\sum_{n, n^{\prime} \in \Delta_{0}}\left\|P_{0, n} f\right\|_{p}^{p / 2}\left\|P_{0, n^{\prime}} g\right\|_{p}^{p / 2}\right)^{2 / p} \\
& \lesssim \max \left\{1, \lambda^{d(1 / 2-2 / p)}\right\} \lambda^{2 / p}\left(\sum_{n}\left\|P_{0, n} f\right\|_{p}^{p}\right)^{1 / p}\left(\sum_{n^{\prime}}\left\|P_{0, n^{\prime}} g\right\|_{p}^{p}\right)^{1 / p} .
\end{aligned}
$$

The asserted bound for $j=0$ follows from (3.7).

Next for $j>0$ we use Lemma 3.3, and thus the assumption $p>2+\frac{4}{d+1}$, and estimate

$$
\left\|S P_{j, n} f S P_{j, n^{\prime}} g\right\|_{L^{p / 2}\left(\mathbb{R}^{d} \times[0, \lambda]\right)} \lesssim 2^{4 j\left(\frac{d}{2}-\frac{d+1}{p}\right)} \lambda^{2 / p}\left\|P_{j, n} f\right\|_{p}\left\|P_{j, n^{\prime}} g\right\|_{p} .
$$

Therefore by (3.6)

$$
\begin{aligned}
& \left\|\widetilde{\mathcal{B}}_{j}[f, g]\right\|_{L^{p / 2}\left(\mathbb{R}^{d} \times[0, \lambda]\right)} \\
& \quad \lesssim \max \left\{1,\left(\lambda^{1 / 2} 2^{-j}\right)^{d(1-4 / p)}\right\} 2^{4 j\left(\frac{d}{2}-\frac{d+1}{p}\right)} \lambda^{2 / p}\left(\sum_{n}\left\|P_{j, n} f\right\|_{p}^{p}\right)^{1 / p}\left(\sum_{n^{\prime}}\left\|P_{j, n^{\prime}} g\right\|_{p}^{p}\right)^{1 / p}
\end{aligned}
$$

and again the asserted bound for $\left\|\widetilde{\mathcal{B}}_{j}[f, g]\right\|_{p / 2}$ follows from (3.7).

\section{Estimates FOR $\exp \left(i t(-\Delta)^{\alpha / 2}\right)$}

We now prove the endpoint estimates of Theorems 1.1 and 1.2. First we remark that by various scaling and symmetry arguments we may assume that $I=[0,1]$.

Consider $\chi_{0}, \chi \in C_{0}^{\infty}(\mathbb{R})$ supported in $(-2,2)$ and $(1 / 2,2)$, respectively, such that

$$
\chi_{0}+\sum_{k \geqslant 1} \chi\left(2^{-k} \cdot\right)=1
$$

We define the operators $T_{k}^{\alpha} \equiv T_{k}$ by

$$
\begin{aligned}
& \left.\widehat{T_{0} \widehat{f(\cdot, t}}\right)(\xi)=\chi_{0}(|\xi|) e^{i t|\xi|^{\alpha}} \widehat{f}(\xi), \\
& \left.\widehat{T_{k} \widehat{f(\cdot, t}}\right)(\xi)=\chi\left(2^{-k}|\xi|\right) e^{i t|\xi|^{\alpha}} \widehat{f}(\xi), \quad k \geqslant 1,
\end{aligned}
$$

so that $U_{t}^{\alpha}=\sum_{k \geqslant 0} T_{k}(\cdot, t)$.

Our main result is the following inequality for vector-valued functions $\left\{f_{k}\right\}_{k=0}^{\infty} \in \ell^{p}\left(L^{p}\right)$.

Theorem 4.1. Let $p \in\left(2+\frac{4}{d+1}, \infty\right), \alpha \neq 1$ and $\beta=\alpha d\left(\frac{1}{2}-\frac{1}{p}\right)-\frac{\alpha}{p}$. Then

$$
\left\|\sum_{k \geqslant 0}\left(\int_{0}^{1}\left|2^{-k \beta} T_{k} f_{k}(\cdot, t)\right|^{p} d t\right)^{1 / p}\right\|_{L^{p}\left(\mathbb{R}^{d}\right)} \lesssim\left(\sum_{k \geqslant 0}\left\|f_{k}\right\|_{p}^{p}\right)^{1 / p} .
$$

The proof will be given in $\S 5$. We now discuss the implications to Theorem 1.1 and 1.2, in fact strengthened versions involving Triebel-Lizorkin spaces $F_{\alpha, q}^{p}$ and Besov spaces $B_{\alpha, q}^{p}$. Here the norms on these spaces are given by the $L^{p}\left(\ell^{q}\right)$ and $\ell^{q}\left(L^{p}\right)$ norms (resp.) of the sequence $\left\{2^{k \alpha} L_{k} f\right\}_{k=0}^{\infty}$, with the usual inhomogeneous dyadic frequency composition $I=\sum_{k \geqslant 0} L_{k}$. See [26]. The following corollary is an immediate consequence of Theorem 4.1, by Minkowski's inequality and Fubini's theorem.

Corollary 4.2. Let $p, \alpha, \beta$ be as in Theorem 4.1. Then

$$
\left(\int_{0}^{1}\left\|U_{t}^{\alpha} f\right\|_{F_{0,1}^{p}\left(\mathbb{R}^{d}\right)}^{p} d t\right)^{1 / p} \lesssim\|f\|_{B_{\beta, p}^{p}\left(\mathbb{R}^{d}\right)} .
$$


This implies Theorem 1.2 since for $p \geqslant 2$ the space $B_{\beta, p}^{p} \equiv F_{\beta, p}^{p}$ contains the Sobolev space $L_{\beta}^{p} \equiv F_{\beta, 2}^{p}$, via the embedding $\ell^{2} \hookrightarrow \ell^{p}$ followed by the Littlewood-Paley inequality, and by the same reasoning $F_{0,1}^{p}$ is imbedded in $L^{p} \equiv F_{0,2}^{p}$. We remark that a similar sharp inequality for the wave equation is proved in [15], in sufficiently high dimensions.

Another consequence of Theorem 4.1 is

Corollary 4.3. Let $p, \alpha$, be as in Theorem 4.1. Let $t \mapsto \vartheta(t)$ be smooth and compactly supported. Then

$$
\|\| \vartheta(\cdot) U_{(\cdot)}^{\alpha} g\left\|_{B_{1 / p, 1}^{p}(\mathbb{R})}\right\|_{L^{p}\left(\mathbb{R}^{d}\right)} \lesssim\|g\|_{B_{\gamma, p}^{p}\left(\mathbb{R}^{d}\right)}, \quad \gamma=\alpha d(1 / 2-1 / p) .
$$

Theorem 1.1 is an immediate consequence of Corollary 4.3 since the Besov space $B_{1 / p, 1}^{p}(\mathbb{R})$ is continuously embedded in the space $C^{0}$ of continuous bounded functions which vanish at infinity.

To see how Corollary 4.3 follows from Theorem 4.1 we introduce dyadic frequency cutoffs in the $t$ variable. We decompose the identity as $I=\sum_{j=0} \mathcal{L}_{j}$ where $\widehat{\mathcal{L}_{j} f}(\tau)=\widetilde{\chi}_{j}(\tau) \widehat{f}(\tau)$ where $\tilde{\chi}_{j}=\tilde{\chi}\left(2^{-j}|\cdot|\right)$ for $j \geqslant 1$, with a suitable $\tilde{\chi} \in C_{0}^{\infty}$ supported in $(1 / 2,2)$ and $\tilde{\chi}_{0}$ is smooth and vanishes for $|\tau| \geqslant 2$. Now we apply $L_{j}$ to $\vartheta T_{k} g$. If $2^{j-\alpha k} \notin\left(2^{-10}, 2^{10}\right)$, then we apply an integration by parts in $s$ to terms of the form

$$
\iint \chi\left(2^{-j}|\tau|\right) \chi\left(2^{-k}|\xi|\right) \widehat{g}(\xi) e^{i(\langle x, \xi\rangle+t \tau)} \int \vartheta(s) e^{i s\left(|\xi|^{\alpha}-\tau\right)} d s d \xi d \tau .
$$

One finds that for this range the contribution of $\mathcal{L}_{j}\left[\vartheta T_{k} g\right]$ is negligible; namely

$$
\left(\int_{\mathbb{R}} \int_{\mathbb{R}^{d}}\left|\mathcal{L}_{j}\left[\vartheta T_{k} g\right](x, s)\right|^{p} d x d s\right)^{1 / p} \lesssim C_{N} \min \left\{2^{-\alpha k N}, 2^{-j N}\right\}\|g\|_{p} \text { if } 2^{j-\alpha k} \notin\left(2^{-10}, 2^{10}\right) .
$$

Thus a localization in $\xi$ where $|\xi| \approx 2^{k}$ corresponds to a localization in $\tau$ where $|\tau| \approx 2^{k \alpha}$. We combine this with Theorem 4.1 applied to $f_{k}=2^{k \beta+k / p} \mathcal{F}^{-1}\left[\chi\left(2^{-k}|\cdot|\right) \widehat{g}\right]$ and obtain

$$
\left\|\sum_{j \geqslant 0} 2^{j / p}\right\| \mathcal{L}_{j}\left[\vartheta U_{(\cdot)}^{\alpha} g\right]\left\|_{L^{p}(\mathbb{R}), d t}\right\|_{L^{p}\left(\mathbb{R}^{d}\right)} \lesssim\left(\sum_{k \geqslant 0} 2^{k \gamma p}\left\|\mathcal{F}^{-1}\left[\chi_{k} \widehat{g}\right]\right\|_{L^{p}\left(\mathbb{R}^{d}\right)}^{p}\right)^{1 / p}
$$

which is (4.2).

\section{Proof of Theorem 4.1}

The localization of the multiplier near the origin $T_{0}$ is easily handled as

$$
\left\|\mathcal{F}^{-1}\left[\chi_{0}(|\cdot|) e^{\left.i t|\cdot|\right|^{\alpha}}\right]\right\|_{L^{1}} \leqslant C
$$

uniformly for $t \in[0,1]$. To see this, since $\mathcal{F}^{-1}\left[\chi_{0}(|\cdot|)\right] \in L^{1}$, it suffices to show that for $\phi$ supported in $(1 / 2,2)$, the $L^{1}$ norm of $\mathcal{F}^{-1}\left[\chi_{0}\left(e^{i t|\cdot|^{\alpha}}-1\right) \phi\left(2^{k}|\cdot|\right)\right]$ is $O\left(2^{-\alpha k}\right)$ for $k \geqslant 0$. But by scaling this follows from showing that the $L^{1}$ norm of $\mathcal{F}^{-1}\left[\chi_{0}\left(2^{-k} \cdot\right)\left(e^{\left.i t 2^{-\alpha k} \cdot\right|^{\alpha}}-1\right) \phi(|\cdot|)\right]$ is $O\left(2^{-\alpha k}\right)$ which follows from the standard Bernstein criterion.

Now, by scaling and Proposition 3.1 with $\lambda \approx 2^{\alpha k}, \mathcal{U}=\{\xi: 1 / 2<|\xi|<2\}$ and $\phi(\xi)=|\xi|^{\alpha}$, we have already proven the estimates

$$
\left\|T_{k} f\right\|_{L^{p}\left(\mathbb{R}^{d} \times[0,1]\right)} \lesssim 2^{k \beta}\|f\|_{L^{p}\left(\mathbb{R}^{d}\right)}, \quad \beta \geqslant \beta(p):=\alpha d\left(\frac{1}{2}-\frac{1}{p}\right)-\frac{\alpha}{p}
$$

for $k>0$ and $p>2+\frac{4}{d+1}$. 
It suffices thus to show that if (5.1) holds for all $k>0$ and all $p>q$, then (4.1) holds for all $p \in(q, \infty)$. Due to our restriction on (5.1) we let $q=2+\frac{4}{d+1}$ and fix $2+\frac{4}{d+1}<r<p$. We can make the additional assumption that the $k$ sum on the left hand side is extended over a finite set (with the constant in the inequality independent of this assumption); the general case then follows by the monotone convergence theorem.

For later reference we state a Sobolev inequality which is proved linking frequency decompositions in $\xi$ and $\tau$ and Young's inequality (just as in the argument used in $\S 4$ to deduce Corollary 4.3 from Theorem 4.1). Namely

$$
\left\|T_{k} f(x, \cdot)\right\|_{L_{t}^{q}[0,1]} \lesssim 2^{\alpha k\left(\frac{1}{p}-\frac{1}{q}\right)}\left\|T_{k} f(x, \cdot)\right\|_{L_{t}^{p}[0,1]} \cdot
$$

holds for $p \leqslant q \leqslant \infty$ (including the endpoint). Alternatively one can also apply the fundamental theorem of calculus to $\left|T_{k} f(x, \cdot)\right|^{q}$ (see e.g. [23]) to get (5.2) for $q=\infty$ and the general inequality follows by convexity.

The main ingredient in the proof of (4.1) (besides (5.1)) will be the Fefferman-Stein sharp function [10] and their inequality

$$
\|F\|_{p} \lesssim\left\|F^{\#}\right\|_{p}
$$

where $p \in(1, \infty)$ and a priori $F \in L^{p}$. We apply this to $\sum_{k>0} 2^{-k \beta(p)}\left\|T_{k} f_{k}(x, \cdot)\right\|_{L_{t}^{p}[0,1]}$ and by (5.1) this function is a priori in $L^{p}$ as the sum in $k$ is assumed to be finite. Thus it will suffice to prove that

$$
\left\|\sup _{x \in Q} \int_{Q}\left|\sum_{k>0} 2^{-k \beta(p)}\left\|T_{k} f_{k}(y, \cdot)\right\|_{L_{t}^{p}[0,1]}-\int_{Q} \sum_{k>0} 2^{-k \beta(p)}\left\|T_{k} f_{k}(z, \cdot)\right\|_{L_{t}^{p}[0,1]} d z\right| d y\right\|_{L_{x}^{p}}
$$

is dominated by $C\left(\sum_{k>0}\left\|f_{k}\right\|_{p}^{p}\right)^{1 / p}$. Here the supremum is taken over all cubes containing $x$, and the slashed integral denotes the average $|Q|^{-1} \int_{Q}$. By the triangle inequality the previous bound follows from

$$
\left\|\sup _{x \in Q} \int_{Q} \sum_{k>0} \int_{Q} 2^{-k \beta(p)}\right\| T_{k} f_{k}(y, \cdot)-T_{k} f_{k}(z, \cdot)\left\|_{L_{t}^{p}[0,1]} d z d y\right\|_{L_{x}^{p}} \lesssim\left(\sum_{k}\left\|f_{k}\right\|_{p}^{p}\right)^{1 / p} .
$$

Denoting the sidelength of $Q$ by $\ell(Q)$, we observe that, by Minkowski's inequality, this would follow from the inequalities

$$
\begin{gathered}
\left\|\sup _{x \in Q} \int_{Q} \sum_{2^{k} \ell(Q) \leqslant 1} \int_{Q} 2^{-k \beta(p)}\right\| T_{k} f_{k}(y, \cdot)-T_{k} f_{k}(z, \cdot)\left\|_{L_{t}^{p}[0,1]} d z d y\right\|_{L_{x}^{p}} \lesssim\left(\sum_{k}\left\|f_{k}\right\|_{p}^{p}\right)^{1 / p} \\
\quad\left\|\sup _{x \in Q} \int_{Q_{2^{k} \ell(Q)>2^{\alpha k}}} \sum^{-k \beta(p)}\right\| T_{k} f_{k}(y, \cdot)\left\|_{L_{t}^{p}[0,1]} d y\right\|_{L_{x}^{p}} \lesssim\left(\sum_{k}\left\|f_{k}\right\|_{p}^{p}\right)^{1 / p} .
\end{gathered}
$$

and

$$
\left\|\sup _{x \in Q} \int_{Q} \sum_{2^{\alpha k} \geqslant 2^{k} \ell(Q)>1} 2^{-k \beta(p)}\right\| T_{k} f_{k}(y, \cdot)\left\|_{L_{t}^{p}[0,1]} d y\right\|_{L_{x}^{p}} \lesssim\left(\sum_{k}\left\|f_{k}\right\|_{p}^{p}\right)^{1 / p} .
$$

First we handle (5.3) and (5.4) by standard estimates and then prove the more interesting inequality (5.5). 
Proof of (5.3). It is enough to consider cubes $Q$ of diameter $\approx 2^{j}$ with $x, y, z \in Q$ and $j+k \leqslant 0$. Let $H_{k}=\mathcal{F}^{-1}\left[\widetilde{\chi}\left(2^{-k}|\cdot|\right)\right]$; then

$$
\left|\nabla H_{k}(w)\right| \lesssim 2^{k} \frac{2^{k d}}{\left(1+2^{k}|w|\right)^{2 N}}
$$

with large $N \geqslant 10 d$. Thus

$$
\begin{aligned}
T_{k} f_{k}(y, t)-T_{k} f_{k}(z, t) & =\int\left[H_{k}(y-w)-H_{k}(z-w)\right] T_{k} f_{k}(w, t) d w \\
& =\iint_{0}^{1}\left\langle(y-z), \nabla H_{k}(z+s(y-z)-w) T_{k} f_{k}(w, t)\right\rangle d s d w
\end{aligned}
$$

which is controlled by a constant multiple of

$$
2^{j+k} \int \frac{2^{k d}}{\left(1+2^{k}|x-w|\right)^{N}}\left|T_{k} f_{k}(w, t)\right| d w .
$$

Thus, using the embedding $\ell^{p} \hookrightarrow \ell^{\infty}$, the right hand side of (5.3) is bounded by

$$
\begin{aligned}
& \left\|\left(\sum_{j}\left|\sum_{0<k \leqslant-j}\left\|2^{j+k} \int \frac{2^{k d}}{\left(1+2^{k}|\cdot-w|\right)^{N}} 2^{-k \beta(p)}\left|T_{k} f_{k}(w, \cdot)\right| d w\right\|_{L_{t}^{p}[0,1]}\right|^{p}\right)^{1 / p}\right\|_{L_{x}^{p}} \\
\lesssim & \sum_{n \geqslant 0} 2^{-n}\left(\sum_{j<-n}\left\|\int \frac{2^{-(n+j)(d-\beta(p))}}{\left(1+2^{-(n+j)}|\cdot-w|\right)^{N}}\left|T_{-(n+j)} f_{-(n+j)}(w, \cdot)\right| d w\right\|_{L^{p}\left(\mathbb{R}^{d} \times[0,1]\right)}^{p}\right)^{1 / p} \\
\lesssim & \sum_{n \geqslant 0} 2^{-n}\left(\sum_{j<-n}\left\|2^{(n+j) \beta(p)} T_{-(n+j)} f_{-(n+j)}\right\|_{L^{p}\left(\mathbb{R}^{d} \times[0,1]\right)}^{p}\right)^{1 / p} \cdot
\end{aligned}
$$

By (5.1) the last expression is dominated by a constant times

$$
\sum_{n \geqslant 0} 2^{-n}\left(\sum_{j<-n}\left\|f_{-(n+j)}\right\|_{p}^{p}\right)^{1 / p} \lesssim\left(\sum_{k}\left\|f_{k}\right\|_{p}^{p}\right)^{1 / p}
$$

and (5.3) is proved.

Proof of (5.4). For a fixed $t$, the operator $T_{k}$ has convolution kernel $K_{k}^{t}$ given by

$$
K_{k}^{t}(x)=\frac{2^{k d}}{(2 \pi)^{d}} \int_{\mathbb{R}^{d}} \chi(|\xi|) e^{i\left(2^{k}\langle x, \xi\rangle+2^{\alpha k} t|\xi|^{\alpha}\right)} d \xi .
$$

Let $C(\alpha)=1$ if $\alpha \in(0,1)$ and let $C(\alpha)=\alpha 2^{\alpha-1}$ if $\alpha \in(1, \infty)$, and define

$$
\mathfrak{B}_{k}(\alpha)=\left\{x:|x| \leqslant 4 C(\alpha) 2^{k(\alpha-1)}\right\} .
$$

Integration by parts yields favorable bounds in the complement of this ball. Observe that

$$
\left|\nabla_{\xi}\left(2^{k}\langle x, \xi\rangle+2^{\alpha k} t|\xi|^{\alpha}\right)\right| \geqslant c_{\alpha} 2^{k}|x| \text { if } x \notin \mathfrak{B}_{k}(\alpha), \quad t \in[0,1],
$$

and we obtain

$$
\left|K_{k}^{t}(x)\right| \leqslant C_{N} 2^{k d}\left(1+2^{k}|x|\right)^{-N} \text { if } x \notin \mathfrak{B}_{k}(\alpha), \quad t \in[0,1] .
$$

Consequently the main contribution of $K_{k}^{t}(x)$ comes when $|x| \leqslant 4 C(\alpha) 2^{k(\alpha-1)}$.

We prove the estimate (5.4) by interpolation between

$$
\left\|\sup _{x \in Q} \int_{Q} \sum_{2^{k} \ell(Q)>2^{\alpha k}} 2^{-k \beta(p)}\right\| T_{k} f_{k}(y, \cdot)\left\|_{L_{t}^{p}[0,1]} d y\right\|_{\infty} \lesssim \sup _{k}\left\|f_{k}\right\|_{\infty}
$$


and

$$
\left\|\sup _{x \in Q} \int_{Q} \sum_{2^{k} \ell(Q)>2^{\alpha k}} 2^{-k \beta(p)}\right\| T_{k} f_{k}(y, \cdot)\left\|_{L_{t}^{p}[0,1]} d y\right\|_{r} \lesssim\left(\sum_{k}\left\|f_{k}\right\|_{r}^{r}\right)^{1 / r},
$$

where $2+\frac{4}{d+1}<r<p$.

Now, as $\beta(p)-\alpha\left(\frac{1}{r}-\frac{1}{p}\right)>\beta(r)$, the $L^{r}$ bound is proven by an application of (5.2), Hölder in $k$, followed by the inequality

$$
\left\|\sup _{x \in Q} \int_{Q}\left(\sum_{k} 2^{-k \beta(r) r}\left\|T_{k} f_{k}(y, \cdot)\right\|_{L_{t}^{r}[0,1]}^{r}\right)^{1 / r} d y\right\|_{r} \lesssim\left(\sum_{k}\left\|f_{k}\right\|_{r}^{r}\right)^{1 / r} .
$$

This is a consequence of the $L^{r}$-boundedness of the Hardy-Littlewood maximal operator, the interchange of the spatial integral and the sum, followed by Fubini and the estimate (5.1) (for the admissible exponent $r>2+4 /(d+1)$ ).

To prove the $L^{\infty}$ bound, we let $Q^{*}$ be a cube with the same center as $Q$ satisfying $\ell\left(Q^{*}\right)=10 d C(\alpha) \ell(Q)$. By Minkowski's inequality it will suffice to prove that

$$
\int_{Q} \sum_{2^{k} \ell(Q)>2^{\alpha k}} 2^{-k \beta(p)}\left\|T_{k}\left[f_{k} \chi_{Q^{*}}\right](y, \cdot)\right\|_{L_{t}^{p}[0,1]} d y \lesssim \sup _{k}\left\|f_{k}\right\|_{\infty}
$$

and

$$
\int_{Q} \sum_{2^{k} \ell(Q)>2^{\alpha k}} 2^{-k \beta(p)}\left\|T_{k}\left[f_{k} \chi_{\mathbb{R}^{d} \backslash Q^{*}}\right](y, \cdot)\right\|_{L_{t}^{p}[0,1]} d y \lesssim \sup _{k}\left\|f_{k}\right\|_{\infty}
$$

uniformly in $Q$.

To prove (5.7), again we apply (5.2) and Hölder a number of times;

$$
\begin{aligned}
& \int_{Q} \sum_{k} 2^{-k \beta(p)}\left\|T_{k}\left[f_{k} \chi_{Q^{*}}\right](y, \cdot)\right\|_{L_{t}^{p}[0,1]} d y \\
& \lesssim|Q|^{-1 / r} \sum_{k} 2^{-k\left(\beta(p)-\alpha\left(\frac{1}{r}-\frac{1}{p}\right)\right)}\left(\int\left\|T_{k}\left[f_{k} \chi_{Q^{*}}\right](y, \cdot)\right\|_{L_{t}^{r}[0,1]}^{r} d y\right)^{1 / r} \\
& \lesssim \sup _{k}|Q|^{-1 / r} 2^{-k \beta(r)}\left(\int\left\|T_{k}\left[f_{k} \chi_{Q^{*}}\right](y, \cdot)\right\|_{L_{t}^{r}[0,1]}^{r} d y\right)^{1 / r} \\
& \lesssim \sup _{k}|Q|^{-1 / r}\left(\int\left|f_{k} \chi_{Q^{*}}\right|^{r} d x\right)^{1 / r} \lesssim \sup _{k}\left\|f_{k}\right\|_{\infty},
\end{aligned}
$$

where the third inequality holds again by the $L^{r}$ version of (5.1).

For (5.8), we note that as $\ell(Q)>2^{k(\alpha-1)}$, and the function is supported in the complement of $Q^{*}$ we can use the rapid decay in formula (5.6). We have that

$$
\begin{aligned}
& \int_{Q} \sum_{2^{k} \ell(Q)>2^{\alpha k}} 2^{-k \beta(p)}\left\|T_{k}\left[f_{k} \chi_{\mathbb{R}^{d} \backslash Q^{*}}\right](y, \cdot)\right\|_{L_{t}^{p}[0,1]} d y \\
\lesssim & \sup _{k} \int_{Q}\left\|\int \frac{2^{k d}}{\left(1+2^{k}|y-z|\right)^{2 d}}\left|f_{k}(z)\right| d z\right\|_{L_{t}^{p}[0,1]} d y \\
\lesssim & \sup _{k}\left\|\int \frac{2^{k d}}{\left(1+2^{k}|\cdot-z|\right)^{2 d}}\left|f_{k}(z)\right| d z\right\|_{\infty} \lesssim \sup _{k}\left\|f_{k}\right\|_{\infty} .
\end{aligned}
$$

This concludes the proof of (5.4) 
Proof of (5.5). We let $\zeta_{j}(x)=\left(d 2^{j}\right)^{-d}$ if $|x| \leqslant d 2^{j}$ and $\zeta_{j}(x)=0$ if $|x| \geqslant d 2^{j}$. Replacing cubes by dyadic balls we see that (5.5) follows from

$$
\left\|\sup _{j} \zeta_{j} * \sum_{\substack{k+j>0 \\(\alpha-1) k \geqslant j}} 2^{-k \beta(p)}\right\| T_{k} f_{k}\left\|_{L_{t}^{p}[0,1]}\right\|_{L_{x}^{p}} \lesssim\left(\sum_{k}\left\|f_{k}\right\|_{p}^{p}\right)^{1 / p} .
$$

Now, for fixed $k$ we cover $\mathbb{R}^{d}$ by a grid $\mathcal{R}_{k}^{\alpha-1}$ consisting of cubes of sidelength $2^{k(\alpha-1)}$. For each $R \in \mathcal{R}_{k}^{\alpha-1}$ let $R^{*}$ be the cube with same center as $R$ and sidelength $C(\alpha) 2^{k(\alpha-1)+10 d}$ where $C(\alpha)$ is as in the proof of (5.4)

For $R \in \mathcal{R}_{k}^{\alpha-1}$ we let $f_{k}^{R}=\chi_{R} f_{k}$. We may then split the left hand side of (5.9) as $I+I I$ where

$$
I=\left\|\sup _{j} \zeta_{j} *\left[\sum_{\substack{k+j>0 \\(\alpha-1) k \geqslant j}} 2^{-k \beta(p)}\left\|\sum_{R \in \mathcal{R}_{k}^{\alpha-1}} \chi_{R^{*}} T_{k} f_{k}^{R}\right\|_{L_{t}^{p}[0,1]}\right]\right\|_{L_{x}^{p}}
$$

and $I I$ is the analogous expression where $\chi_{R^{*}}$ is replaced with $\chi_{\mathbb{R}^{d} \backslash R^{*}}$.

By Hardy-Littlewood, Minkowski, Fubini, (5.6), and Young's inequality, we dominate

$$
\begin{aligned}
I I & \lesssim \sum_{k \geqslant 0} 2^{-k \beta(p)}\left\|\sum_{R \in \mathcal{R}_{k}^{\alpha-1}} \chi_{\mathbb{R}^{d} \backslash R^{*}} T_{k} f_{k}^{R}\right\|_{L^{p}\left(\mathbb{R}^{d} \times[0,1]\right)} \\
& \lesssim \sum_{k \geqslant 0} 2^{-k \beta(p)}\left(\int_{0}^{1} \int\left[\int \frac{2^{k d}}{\left(1+2^{k}|x-y|\right)^{2 d}} \sum_{R \in \mathcal{R}_{k}^{\alpha-1}}\left|f_{k}^{R}(y)\right| d y\right]^{p} d x d t\right)^{1 / p} \\
& \lesssim \sum_{k \geqslant 0} 2^{-k \beta(p)}\left\|\sum_{R \in \mathcal{R}_{k}^{\alpha-1}} f_{k}^{R}\right\|_{p} \lesssim \sup _{k}\left\|f_{k}\right\|_{p} \lesssim\left(\sum_{k}\left\|f_{k}\right\|_{p}^{p}\right)^{1 / p} .
\end{aligned}
$$

Concerning the main term $I$ we use the imbedding $\ell^{p} \hookrightarrow \ell^{\infty}$, interchange a sum and an integral, and apply Minkowski's inequality, so that

$$
I \lesssim\left(\sum_{j}\left\|\zeta_{j} *\left[\sum_{\substack{k+j>0 \\(\alpha-1) k \geqslant j}} 2^{-k \beta(p)} \sum_{R \in \mathcal{R}_{k}^{\alpha-1}} \chi_{R^{*}}\left\|T_{k} f_{k}^{R}\right\|_{L_{t}^{p}[0,1]}\right]\right\|_{L_{x}^{p}}^{p}\right)^{1 / p} .
$$

Now for $R \in \mathcal{R}_{k}^{\alpha-1}, R^{*}$ has sidelength greater than $2^{j}$, so for fixed $k$ the functions $\zeta_{j} * \chi_{R^{*}}$ have bounded overlap, uniformly in $k$. Setting $n=k+j>0$ and applying Minkowski's inequality, we get

$$
I \lesssim \sum_{n>0} I_{n}
$$

where

$$
I_{n}=\left(\sum_{j<n} \sum_{R \in \mathcal{R}_{n-j}^{\alpha-1}} 2^{-(n-j) \beta(p) p}\left\|\zeta_{j} *\right\| T_{n-j} f_{n-j}^{R}\left\|_{L_{t}^{p}[0,1]}\right\|_{L_{x}^{p}}^{p}\right)^{1 / p} .
$$

As before choose $r$ so that $2+\frac{4}{d+1}<r<p$. It will suffice to show that

$$
I_{n} \lesssim 2^{-n d\left(\frac{1}{r}-\frac{1}{p}\right)}\left(\sum_{k}\left\|f_{k}\right\|_{p}^{p}\right)^{1 / p} \text {. }
$$

Observe that by Young's inequality the convolution with $\zeta_{j}$ maps $L^{r}\left(\mathbb{R}^{d}\right)$ to $L^{p}\left(\mathbb{R}^{d}\right)$ with operator norm $O\left(2^{-j d(1 / r-1 / p)}\right)$. Moreover by (5.2) we have

$$
\left\|T_{n-j} f_{n-j}^{R}(x, \cdot)\right\|_{L_{t}^{p}[0,1]} \lesssim 2^{(n-j) \alpha\left(\frac{1}{r}-\frac{1}{p}\right)}\left\|T_{n-j} f_{n-j}^{R}(x, \cdot)\right\|_{L_{t}^{r}[0,1]} .
$$


Thus we can bound

$$
I_{n} \lesssim\left(\sum_{j} 2^{-j d\left(\frac{1}{r}-\frac{1}{p}\right) p} 2^{(n-j) \alpha\left(\frac{1}{r}-\frac{1}{p}\right) p} 2^{-(n-j) \beta(p) p} \sum_{R \in \mathcal{R}_{n-j}^{\alpha-1}}\left\|T_{n-j} f_{n-j}^{R}\right\|_{L^{r}\left(\mathbb{R}^{d} \times[0,1]\right)}^{p}\right)^{\frac{1}{p}}
$$

which, by (5.1), is

$$
\lesssim\left(\sum_{j} 2^{-j d\left(\frac{1}{r}-\frac{1}{p}\right) p} 2^{(n-j) \alpha\left(\frac{1}{r}-\frac{1}{p}\right) p} 2^{-(n-j) \beta(p) p} \sum_{R \in \mathcal{R}_{n-j}^{\alpha-1}} 2^{(n-j) \beta(r) p}\left\|f_{n-j}^{R}\right\|_{r}^{p}\right)^{\frac{1}{p}} .
$$

Since $f_{n-j}^{R}$ is supported on the cube $R$ of size $2^{(n-j)(\alpha-1) d}$ we see by Hölder's inequality that the last displayed expression is dominated by a constant times

$$
\left(\sum_{j} 2^{-j d\left(\frac{1}{r}-\frac{1}{p}\right) p} 2^{(n-j) \alpha\left(\frac{1}{r}-\frac{1}{p}\right) p} 2^{-(n-j) \beta(p) p} 2^{(n-j) \beta(r) p} 2^{(n-j)(\alpha-1) d\left(\frac{1}{r}-\frac{1}{p}\right) p} \sum_{R \in \mathcal{R}_{n-j}^{\alpha-1}}\left\|f_{n-j}^{R}\right\|_{p}^{p}\right)^{\frac{1}{p}} .
$$

Now this simplifies, after summation in $R$, to

$$
I_{n} \lesssim 2^{-n d\left(\frac{1}{r}-\frac{1}{p}\right)}\left(\sum_{j}\left\|f_{n-j}\right\|_{p}^{p}\right)^{\frac{1}{p}} \leqslant C 2^{-n d\left(\frac{1}{r}-\frac{1}{p}\right)}\left(\sum_{k}\left\|f_{k}\right\|_{p}^{p}\right)^{1 / p} .
$$

This finishes the proof of (5.10) and thereby (5.5) and concludes the proof of Theorem 4.1.

Acknowledgement. The first author thanks Gustavo Garrigós, Manuel Portilheiro, Ana Vargas and Luis Vega for helpful conversations.

\section{REFERENCES}

[1] A. Baernstein and E.T. Sawyer, Embedding and multiplier theorems for $H^{p}\left(R^{n}\right)$, Mem. Amer. Math. Soc. 53 (1985), no. 318 .

[2] A. Carbery, The boundedness of the maximal Bochner-Riesz operator on $L^{4}\left(R^{2}\right)$, Duke Math. J. 50 (1983), no. 2, 409-416.

[3] _ Restriction implies Bochner-Riesz for paraboloids, Math. Proc. Cambridge Philos. Soc., 111 (1992), 525-529.

[4] L. Carleson, Some analytic problems related to statistical mechanics. Euclidean harmonic analysis (Proc. Sem., Univ. Maryland, College Park, Md., 1979), pp. 5-45, Lecture Notes in Math., 779, Springer, Berlin, 1980.

[5] L. Carleson and P. Sjölin, Oscillatory integrals and a multiplier problem for the disc, Studia Math. 44 (1972), 287-299.

[6] M. Christ, On almost everywhere convergence of Bochner-Riesz means in higher dimensions, Proc. Amer. Math. Soc. 95 (1985), 16-20.

[7] P. Constantin, J.-C. Saut, Local smoothing properties of dispersive equations, J. Amer. Math. Soc. 1 (1988), 413-439.

[8] C. Fefferman, Inequalities for strongly singular convolution operators, Acta Math. 1241970 9-36.

[9] _ _ A note on spherical summation multipliers, Israel J. Math., 15, (1973), 44-52.

[10] C. Fefferman and E.M. Stein, $H^{p}$ spaces of several variables, Acta Math. 129 (1972), no. 3-4, 137-193.

[11] L. Hörmander, Oscillatory integrals and multipliers on $F L^{p}$, Ark. Mat. 11 (1973), 1-11.

[12] S. Lee, Improved bounds for Bochner-Riesz and maximal Bochner-Riesz operators, Duke Math. J. 122 (2004), 205-232.

[13] S. Lee and A. Seeger, manuscript.

[14] A. Miyachi, On some singular Fourier multipliers, J. Fac. Sci. Univ. Tokyo Sect. IA Math., 28 (1981), 267-315.

[15] F. Nazarov and A. Seeger, Radial Fourier multipliers in high dimensions, preprint.

[16] K.M. Rogers, A local smoothing estimate for the Schrödinger equation, Adv. Math. 219 (2008), no. 6, 2105-2122. 
[17] K.M. Rogers and P. Villarroya, Global estimates for the Schrödinger maximal operator, Ann. Acad. Sci. Fenn. Math. 32 (2007), 425-435.

[18] A. Seeger, On quasiradial Fourier multipliers and their maximal functions, J. Reine Angew. Math. 370 (1986), 61-73.

[19] - Remarks on singular convolution operators, Studia Math. 97 (1990), 91-114.

[20] Endpoint inequalities for Bochner-Riesz multipliers in the plane, Pacific J. Math. 174 (1996), 543-553.

[21] P. Sjölin, Regularity of solutions to the Schrödinger equation, Duke Math. J., 55 (1987), 699-715.

[22] C.D. Sogge, Propagation of singularities and maximal functions in the plane, Invent. Math. 104 (1991), no. 2, 349-376.

[23] E. Stein, Harmonic analysis: real-variable methods, orthogonality, and oscillatory integrals, Princeton University Press, (1993).

[24] T. Tao, A Sharp bilinear restriction estimate for paraboloids, Geom. Funct. Anal. 13 (2003), 1359-1384.

[25] T. Tao, A. Vargas and L. Vega. A bilinear approach to the restriction and Kakeya conjectures, J. Amer. Math. Soc. 11 (1998), 967-1000.

[26] H. Triebel, Theory of function spaces. Monographs in Mathematics, 78. Birkhäuser Verlag, Basel, 1983.

[27] L. Vega, Schrödinger equations: pointwise convergence to the initial data, Proc. Amer. Math. Soc. 102 (1988), no. 4, 874-878.

[28] T. Wolff, Local smoothing type estimates on $L^{p}$ for large p, Geom. Funct. Anal. 10 (2000), no. 5, 1237-1288.

[29] A. Zygmund, On Fourier coefficients and transforms of functions of two variables, Studia Math. 50 (1974), 189-201.

Keith Rogers, Instituto de Ciencias Matematicas CSIC-UAM-UC3M-UCM, 28006 Madrid, SPAIN

E-mail address: keith.rogers@uam.es

Andreas Seeger, Department of Mathematics, University of Wisconsin, 480 Lincoln Drive, MAdison, WI, 53706, USA

E-mail address: seeger@math.wisc.edu 